\title{
3D Discrete Dislocation Dynamics: Influence of Segment Mobility on Critical Shear Stress
}

\author{
T. ZÁLEŽÁK* AND A. DLOUHÝ \\ Institute of Physics of Materials, Academy of Sciences (CEITEC IPM), Žižkova 22, 61662 Brno, Czech Republic
}

\begin{abstract}
We use 3D discrete dislocation dynamics technique to study a low-angle tilt boundary migration subjected to applied shear stress at high temperatures, where diffusion significantly contributes to the dislocation motion. The model considers Peach-Koehler forces due to interactions between individual straight dislocation segments. The model also addresses dislocation plasticity in a field of impenetrable incoherent spherical precipitates. Velocities of the individual dislocation segments are calculated in relation to the crystallography of the material. Several calculation series have been carried out for different velocity and driving force relations. The results show that there exists a critical applied shear stress, below which the low angle dislocation boundary cannot surpass the rigid precipitates and remains in an equilibrium configuration. This agrees with experimental results obtained in creep tests of dispersion strengthened alloys. The critical stresses have been calculated also for situations where the applied stress was decreased during the interaction between the low-angle tilt boundary and the precipitates.
\end{abstract}

DOI: 10.12693 /APhysPolA.128.654

PACS: 61.72.Hh, 61.72.Lk, 62.20.Hg

\section{Introduction}

The motion of dislocations is a key mechanism of crystal plasticity. The 3D discrete dislocation dynamics (DDD) model provides a detailed insight into complex dislocation interactions and thus helps understanding deformation of materials. The 3D DDD model considers diffusion processes [1], which considerably influence high temperature plasticity [2]. We apply the model to a lowangle dislocation boundary migrating in a field of rigid precipitates and we use the results to elucidate creep behaviour of precipitate hardened materials.

\section{Brief description of the model}

The dislocation system is represented by short straight segments arranged into continuous lines. The linear theory of elasticity then allows to compute a local stress field as a sum of fields originating from all individual segments and, optionally, the externally applied stress [3]. At the centres of dislocation segments the local stress fields generate the Peach-Koehler forces (PKFs). The velocity of a dislocation segment is given by a linear function of the PKF and a diffusion factor $[1,3]$ :

$$
\beta(b)=\frac{D_{0} \Omega}{b^{2} k T} \exp \left(-\frac{Q}{R T}\right),
$$

where $\Omega$ is an atomic volume, $b$ is the length of Burgers vector, $k$ is Boltzmann constant, $R$ is gas constant, $T$ is temperature, $D_{\mathrm{s}}=D_{0} \exp (-Q / R T)$ is the factor of selfdiffusion and $Q$ is the activation energy of self-diffusion. The following velocity relations have been used:

\footnotetext{
* corresponding author; e-mail: zalezak@ipm.cz
}

\subsection{Force decomposition according to material crystallography, regular bcc or fcc slip systems}

For each dislocation segment with Burgers vector $\boldsymbol{b}$ and a direction $\boldsymbol{\xi}$ we find a slip plane normal and decompose the Peach-Koehler force $\boldsymbol{F}$ to a climb $\boldsymbol{F}_{\mathrm{C}}$ and a glide part $\boldsymbol{F}_{\mathrm{G}}$ :

$$
\boldsymbol{n}=\frac{\boldsymbol{b} \times \boldsymbol{\xi}}{|\boldsymbol{b} \times \boldsymbol{\xi}|}, \quad \boldsymbol{F}_{\mathrm{C}}=(\boldsymbol{F} \cdot \boldsymbol{n}) \boldsymbol{n}, \quad \boldsymbol{F}_{\mathrm{G}}=\boldsymbol{F}-\boldsymbol{F}_{\mathrm{C}} .
$$

If the slip plane normal $\boldsymbol{n}$ matches any of the $\boldsymbol{n}_{\mathrm{c}} \in N_{\mathrm{c}}$, where $N_{\mathrm{c}}$ denotes the set of regular slip plane normals, we take $\boldsymbol{F}_{\mathrm{G}}$ and compute

$$
\boldsymbol{F}_{\mathrm{G}_{2}}=\left(\boldsymbol{F}_{\mathrm{G}} \cdot \boldsymbol{n}_{\mathrm{c}}\right) \boldsymbol{n}_{\mathrm{c}}, \quad \boldsymbol{F}_{\mathrm{G}_{1}}=\boldsymbol{F}_{\mathrm{G}}-\boldsymbol{F}_{\mathrm{G}_{2}} .
$$

Otherwise, we set $\boldsymbol{F}_{\mathrm{G}_{1}}=\mathbf{0}$ and $\boldsymbol{F}_{\mathrm{G}_{2}}=\boldsymbol{F}_{\mathrm{G}}$. The final relation for segment velocity is

$$
\boldsymbol{v}=a_{\mathrm{G}} \beta(b) \boldsymbol{F}_{\mathrm{G}_{1}}+a_{\mathrm{NC}} \beta(b) \boldsymbol{F}_{\mathrm{G}_{2}}+a_{\mathrm{C}} \beta(b) \boldsymbol{F}_{\mathrm{C}} .
$$

We choose $a_{\mathrm{G}}=10, a_{\mathrm{C}}=1$ and $a_{\mathrm{NC}} \in\{1,2\}$. The glide in low-index crystallographic planes is much faster than non-compact glide, which is comparable to the climb motion.

Based on our previous work [4], we know that the particular variations in the $a_{\mathrm{G}}$ as high as one or two orders of magnitude have only minor influence on the calculated threshold stress.

\subsection{Force decomposition according to crystallography and edge/screw character of the segment}

At first, we decompose the dislocation with a Burgers vector $\boldsymbol{b}$ to a pure edge and a pure screw component with Burgers vectors $\boldsymbol{b}_{\mathrm{e}}$ and $\boldsymbol{b}_{\mathrm{s}}$, respectively. The PKFs $\boldsymbol{F}_{\mathrm{e}}$ and $\boldsymbol{F}_{\mathrm{s}}$ are then evaluated separately for the pure edge and screw components. Further, $\boldsymbol{F}_{\mathrm{e}}$ is decomposed into a glide component $\boldsymbol{F}_{\mathrm{e}, \mathrm{G}}$ and a climb component $\boldsymbol{F}_{\mathrm{e}, \mathrm{C}}$ (see Eq. (2)) with respect to the slip plane of the dislocation segment. If both edge force component $\boldsymbol{F}_{\mathrm{e}}$ and the dislocation segment lie in a crystallographic slip plane, 
we set the edge glide factor to $A_{\mathrm{e}, \mathrm{G}}=a_{\mathrm{G}} \beta\left(\left|\boldsymbol{b}_{\mathrm{e}}\right|\right)$, otherwise we set $A_{\mathrm{e}, \mathrm{G}}=a_{\mathrm{NC}} \beta\left(\left|\boldsymbol{b}_{\mathrm{e}}\right|\right)$. The edge climb factor is always $A_{\mathrm{e}, \mathrm{C}}=a_{\mathrm{C}} \beta\left(\left|\boldsymbol{b}_{\mathrm{e}}\right|\right)$. If the dislocation segment and the screw force component lie in a crystallographic slip plane, we set the screw factor to $A_{\mathrm{s}}=a_{\mathrm{G}} \beta\left(\left|\boldsymbol{b}_{\mathrm{s}}\right|\right)$, otherwise we set $A_{\mathrm{s}}=a_{\mathrm{NC}} \beta\left(\left|\boldsymbol{b}_{\mathrm{s}}\right|\right)$. Finally, the total velocity of the dislocation segment is

$$
\boldsymbol{v}=\left(\frac{b_{\mathrm{s}}}{b}\right)^{2} A_{\mathrm{s}} \boldsymbol{F}_{\mathrm{s}}+\left(\frac{b_{\mathrm{e}}}{b}\right)^{2}\left(A_{\mathrm{e}, \mathrm{G}} \boldsymbol{F}_{\mathrm{e}, \mathrm{G}}+A_{\mathrm{e}, \mathrm{C}} \boldsymbol{F}_{\mathrm{e}, \mathrm{C}}\right) .
$$

\subsection{Force decomposition according to crystallography} and edge/screw character of the segment, where the dislocations encounter reaction forces from the precipitates

The former kinetic relations do not consider any force contributions originating in the precipitates. The dislocations cannot pass through the matrix-particle interface (surface of the rigid incoherent precipitates) and remain pinned on the boundary, if the driving force is directed inside the precipitate. However, if we add a reaction force which just compensates that part of the driving force pushing the dislocation to the phase boundary (see Fig. 1), the final dislocation velocity will be tangent to the phase boundary and its magnitude will be equal to

$$
\begin{aligned}
& v=v_{\mathrm{s}}+v_{\mathrm{e}}, \quad v_{\mathrm{s}}=\left(\frac{b_{\mathrm{s}}}{b}\right)^{2} A_{\mathrm{s}} F_{\mathrm{s}} \cos \vartheta, \\
& v_{\mathrm{e}}=\frac{F_{\mathrm{e}} \cos \varphi}{\frac{\cos ^{2} \alpha}{\left(b_{\mathrm{e}} / b\right)^{2} A_{g}}+\frac{\sin ^{2} \alpha}{\left(b_{\mathrm{e}} / b\right)^{2} A_{\mathrm{c}}} .}
\end{aligned}
$$

For further details of the model, see $[5,6]$.

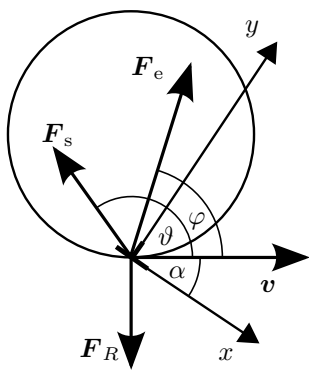

Fig. 1. Reaction force.

\section{Application to low angle tilt boundaries}

Three distinct velocity relations in our 3D DDD model have been evaluated for a dislocation system composing a low angle tilt boundary (LATB). The system evolves under an action of an externally applied stress $\sigma_{x z}$. The dislocation system is represented by a simulation cell with two planes of symmetry $Y=0$ and $Z=0$ with $n$ equidistant parallel edge dislocation lines in a starting position. The simulation cell is replicated in a $3 \times 3$ pattern to approximate periodicity along $Y$ and $Z$ axes. The material and geometry parameters are summarized in the Table.

Initially, the local internal stresses do not affect the planar shape of the tilt boundary due to the periodic

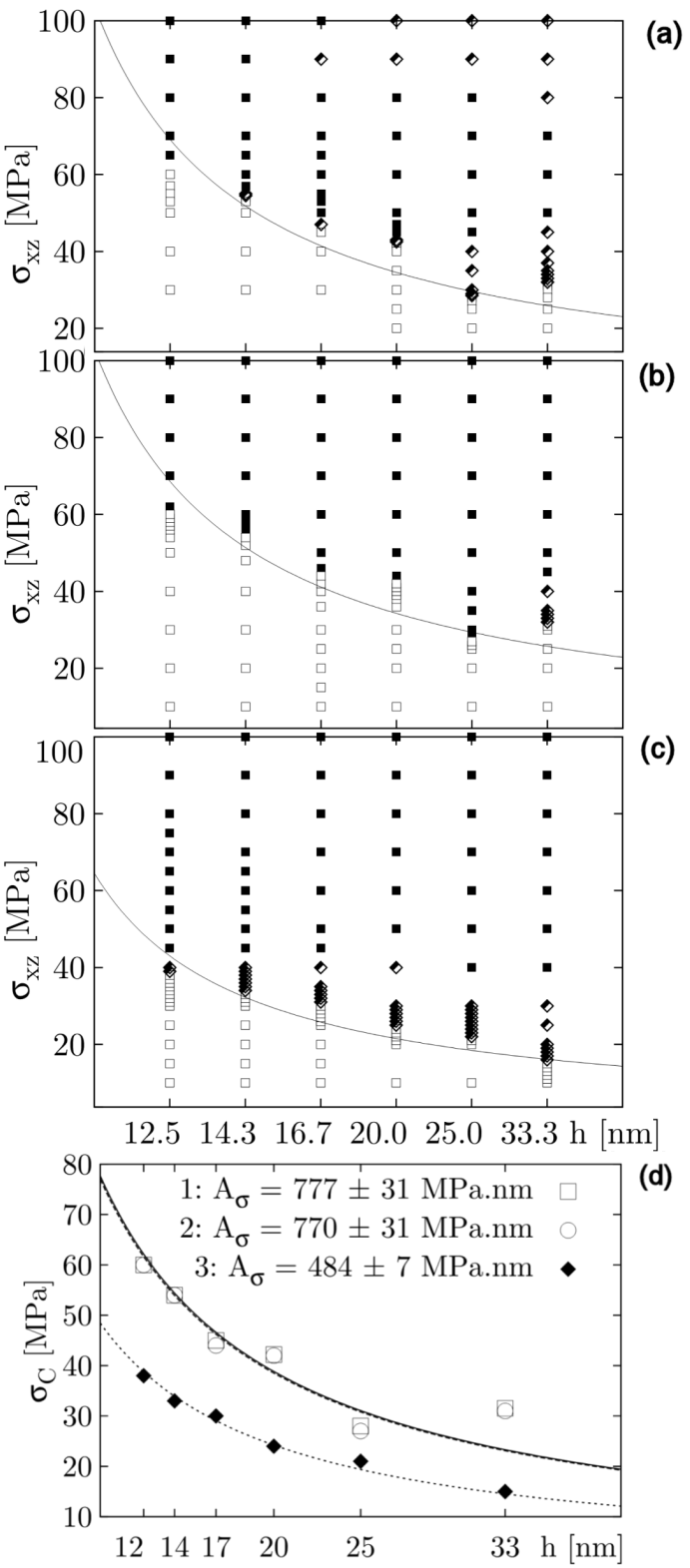

Fig. 2. (a,b,c) Evolution regimes of the tilt boundary for velocity relations $1-3: \square-$ mode $S T O P, \mathbf{\square}-$ mode $P A S S, \diamond$ - mode PASS \&S SPLIT, (d) comparison of critical shear stresses.

boundary conditions. After the tilt boundary encounters rigid precipitates, the evolution can continue in three distinct regimes $[5,6]$. For low applied stresses and/or high initial dislocation densities, the boundary movement ceases and the dislocations remain pinned by the precipitates (mode STOP). For high applied stresses and/or low initial dislocation densities, the boundary either fully overcomes the precipitates (mode PASS), or disintegrates while a major part of the dislocations overcome the precipitates (mode PASS \&S SPLIT). The regimes for various velocity relations are summarized in Fig. $2 \mathrm{a}-\mathrm{c}$.

The mode $S T O P$ is delimited from the modes PASS and PASS \&S SPLIT by a critical shear stress, which 


\section{TABLE}

Input parameters of the model.

\begin{tabular}{c|c|c}
\hline \hline$\mu$ & $80 \mathrm{GPa}$ & shear modulus \\
$\nu$ & 0.3 & the Poisson ratio \\
$D_{0}$ & $2 \mathrm{~cm}^{2} \mathrm{~s}^{-1}$ & diffusion factor \\
$Q$ & $240 \mathrm{~kJ} \mathrm{~mol}^{-1}$ & activation energy \\
$T$ & $873 \mathrm{~K}$ & temperature \\
$\Omega$ & $(0.35 \mathrm{~nm})^{3}$ & atomic volume \\
$\boldsymbol{b}$ & $(0.2,0,0) \mathrm{nm}$ & the Burgers vector \\
$\Delta t$ & $3 \mathrm{~ms}$ & time step \\
$N$ & 32 & \# of segments \\
$n$ & $\{7, \ldots, 17\}$ & $\#$ of lines in a cell \\
$a_{\mathrm{Y}} ; a_{\mathrm{Z}}$ & $200 \mathrm{~nm}$ & cell dimensions \\
$h$ & $a_{\mathrm{Z}} /(n-1)$ & initial line spacing \\
$\boldsymbol{c}$ & {$[-50,0,0] \mathrm{nm}$} & particle center \\
$d$ & $100 \mathrm{~nm}$ & particle diameter \\
$\lambda$ & $200 \mathrm{~nm}$ & particle distance \\
$l$ & $\langle 3,8\rangle \mathrm{nm}$ & segment length
\end{tabular}

can be expressed as a function of initial dislocation line spacing

$$
\sigma_{\mathrm{C}}=A_{\sigma} / h \text {. }
$$

The factors $A_{\sigma}$ listed in Fig. $2 \mathrm{~d}$ have been obtained from calculated data by a least-squares fitting. The results show that the critical shear stress is only weakly sensitive to actual velocity formula, as long as the reaction force of the precipitates is not taken into account. The reaction force greatly facilitates the dislocation movement on the interface between the matrix and the precipitates. However, the critical shear stress is still present, though it is considerably lower than in the former cases (Fig. 2c, d).

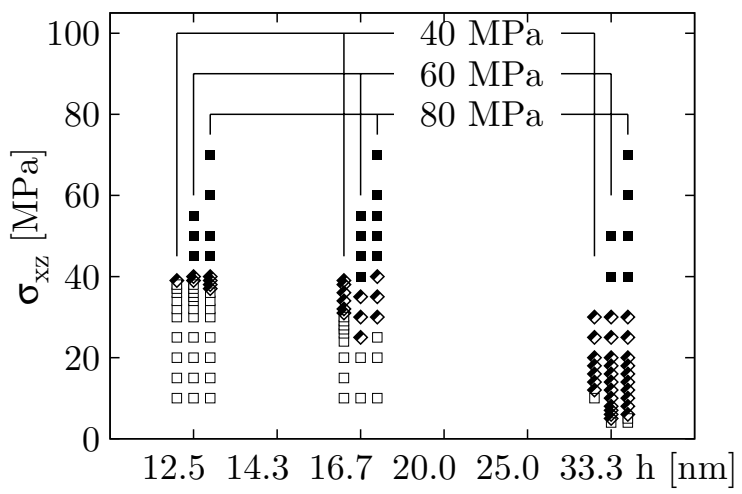

Fig. 3. Evolution regimes of the tilt boundary for $\sigma_{x z}$ lowered from $\sigma_{x z} \in\{40,60,80\} \mathrm{MPa}$ when $\dot{\varepsilon}_{\text {min }}$ had been reached.

The critical shear stress occurs also in case where the applied stress is lowered during the simulation (a numerical stress-dip test). For initial applied stresses $\sigma_{x z} \in\{40,60,80\} \mathrm{MPa}$ and line spacings $h \in$ $\{12.5,16 . \overline{6}, 33 . \overline{3}\} \mathrm{nm}$, the $\sigma_{x z}$ was lowered in integration steps, where the strain rate $\dot{\varepsilon}(t)$ was equal to the minimum strain rate $\dot{\varepsilon}_{\text {min }}$. The evolution regimes are displayed in Fig. 3.

\section{Discussion}

There is considerable body of experimental evidence showing that low angle dislocation boundaries form during high temperature loading. This concerns many materials including dispersion strengthened alloys [7, 8]. Our results show that the migration of LATBs at high temperatures ceases when the applied stress is below critical shear stress even in case when the precipitates exert a reaction force upon dislocation segments. This is in line with numerous creep experiments, where threshold stresses have been observed [7]. The critical shear stress occurs also when the applied stress is lowered during the LATB migration. A significant difference in the two sets of critical stresses has been detected.

\section{Summary and conclusion}

Our 3D discrete dislocation dynamics (DDD) model considers multiple techniques how to calculate the dislocation velocity from the Peach-Koehler forces. The results show that the critical shear stress $\sigma_{\mathrm{C}}$ is only weakly sensitive to the particular technique, except when the reaction forces from precipitates are taken into account. For a particular loading conditions that place the system into the domain PASS, the results of the numerical stressdip tests suggested that the threshold-like behaviour persists for resulting stress levels lower than $\sigma_{\mathrm{C} \text {,dip }}$. However, the values of $\sigma_{\mathrm{C} \text {,dip }}$ were generally lower than the threshold stresses $\sigma_{\mathrm{C}}$ obtained in the calculations with the constant loading conditions.

\section{Acknowledgments}

This work has been supported by the Czech Science Foundation in the project $14-22834 \mathrm{~S}$ and by the Ministry of Education, Youth and Sports in the project No. CZ.1.07/2.3.00/20.0214 (Human resources developments in the research of physical and material properties of emerging, newly developed and applied engineering materials).

\section{References}

[1] D. Mordehai, E. Clouet, M. Fivel, M. Verdier, Philos. Mag. 88, 899 (2008).

[2] J. Čadek, Creep in Metallic Materials, Elsevier, Amsterdam 1988.

[3] P. Hirth, J. Lothe, Theory of Dislocations, 2nd ed., Krieger Publ., Malabar 1992.

[4] D. Holec, A. Dlouhý, Zeitschrift f. Metalkde 96, 558 (2005).

[5] T. Záležak, A. Dlouhý, Acta Phys. Pol. A 122, 450 (2012).

[6] T. Záležak, A. Dlouhý, Key Eng. Mater. 592, 87 (2014).

[7] J.H. Hausselt, W.D. Nix, Acta Metall. 25, 595 (1977).

[8] W. Blum, in: Materials Science and Technology, Vol. 6, Ed. H. Mughrabi, VCH Verlagsgesellschaft, Weinheim 1993. 